BULLETIN (New Series) OF THE

AMERICAN MATHEMATICAL SOCIETY

Volume 41, Number 4, Pages 507-521

S 0273-0979(04)01009-2

Article electronically published on February 9, 2004

\title{
KAM THEORY: THE LEGACY OF KOLMOGOROV'S 1954 PAPER
}

\author{
HENK W. BROER
}

\begin{abstract}
Kolmogorov-Arnold-Moser (or KAM) theory was developed for conservative dynamical systems that are nearly integrable. Integrable systems in their phase space usually contain lots of invariant tori, and KAM theory establishes persistence results for such tori, which carry quasi-periodic motions. We sketch this theory, which begins with Kolmogorov's pioneering work.
\end{abstract}

\section{INTRODUCTION}

At the International Mathematical Congress of 1954, held at Amsterdam, A.N. Kolmogorov gave a closing lecture with the title "The general theory of dynamical systems and classical mechanics" 40], discussing the paper [39. The event took place in the Amsterdam Concertgebouw, and it has played a major role in the development of what is now called Kolmogorov-Arnold-Moser (or KAM) theory.

In this lecture Kolmogorov discusses the occurrence of multi- or quasi-periodic motions, which in the phase space are confined to invariant tori. He restricts himself to conservative (or Hamiltonian) dynamical systems, as these are generally used for modelling in classical mechanics. Invariant (Lagrangean) tori that carry quasi-periodic motions were well-known to occur in Liouville integrable systems, and Kolmogorov's lecture deals with their persistence under small, non-integrable perturbation of the Hamiltonian.

The general message of KAM theory is that, generically, in small perturbations of integrable systems the union of quasi-periodic Lagrangean tori has positive (Liouville) measure both in the phase space and in the energy hypersurfaces. On the one hand this statement applies to many concrete models of classical mechanics. Examples are certain versions of the $n$-body problem, of which the general non-integrability already had been established by Poincaré. Historically its consequences for philosophical issues like the stability of the solar system are of interest. Indeed, perpetual stability of the solar system certainly would be granted if its actual motion were quasi-periodic.

On the other hand, and from a more global point of view, the measure-theoretical part of KAM theory implies that for typical Hamiltonian systems in finitely many degrees of freedom, no ergodicity holds, since the energy hypersurfaces can be decomposed in several disjoint invariant sets of positive measure. This is of particular interest for statistical physics, where the ergodic hypothesis roughly claims that the system, when confined to bounded energy hypersurfaces, is ergodic. This paradox

Received by the editors November 18, 2003, and, in revised form, December 16, 2003.

2000 Mathematics Subject Classification. Primary 37C55, 37C70, 37A60, 34C15.

(C)2004 American Mathematical Society 
probably is resolved as the number of particles is increasing since the obstruction to ergodicity provided by the KAM tori then seems to decrease rapidly in importance.

The present paper sketches certain developments related to the Kolmogorov 1954 lecture [40]. We start with discussing the linearization problem near a fixed point of an analytic map of the complex plane. This is the first known occasion where a small divisor problem was solved, the kind of problem which is so central in KAM theory. Next we turn to the dynamics of circle maps, which goes back to Arnold, followed by a digression to area preserving twist maps, where the name of Moser comes in. After this we discuss conservative KAM theory in greater generality, also touching the ergodic 'paradox'. For an earlier version of the present paper see [11.

Remark. A property is called typical if it occurs on an open set of the dynamical systems at hand. Regarding the topology on 'function space', one may think of a (weak) Whitney topology for differentiable systems or of the compact-open topology for analytic systems. The property we have in mind is the occurrence in phase space of quasi-periodic invariant tori with positive measure. Compare with, e.g., [17], [23].

\section{Complex linearization}

Consider a local holomorphic map (or a germ) $F:(\mathbf{C}, 0) \rightarrow(\mathbf{C}, 0)$ of the form $F(z)=\lambda z+f(z)$ with $f(0)=f^{\prime}(0)=0$. The question is whether there exists a local biholomorphic transformation $\Phi:(\mathbf{C}, 0) \rightarrow(\mathbf{C}, 0)$ such that

$$
\Phi \circ F=\lambda \cdot \Phi .
$$

We say that $\Phi$ linearizes $F$ near its fixed point.

Formal solution. First consider the problem at a formal level. The corresponding results go back to Poincaré; for an overview see Arnold [5]. Given a series expansion $f(z)=\sum_{j \geq 2} f_{j} z^{j}$ we look for another series $\Phi(z)=z+\sum_{j \geq 2} \phi_{j} z^{j}$, such that the conjugation relation (11) holds formally. It turns out that a formal solution exists whenever $\lambda \neq 0$ is not a root of 1 . Indeed, the coefficients $\phi_{j}$ can be determined recursively by the following equations:

$$
\begin{aligned}
\lambda(1-\lambda) \phi_{2} & =f_{2}, \\
\lambda\left(1-\lambda^{2}\right) \phi_{3} & =f_{3}+2 \lambda f_{2} \phi_{2}, \\
\lambda\left(1-\lambda^{n-1}\right) \phi_{n} & =f_{n}+\text { already known terms. }
\end{aligned}
$$

From this the claim directly follows.

Convergence. In the hyperbolic case where $0<|\lambda| \neq 1$ the series for $\Phi$ has positive radius of convergence. This was proven by Poincaré, not by considering the series, but by a direct iteration method; compare [5].

So there remains the elliptic case with $\lambda \in \mathbf{T}^{1}$ (the complex unit circle). We observe from the equations (2) that even if $\lambda$ is not a root of unity, its powers may accumulate on 1 . This would give small divisors in the formal series of $\Phi$, which casts doubt on its convergence.

This problem was successfully solved by C.L. Siegel [65] in 1942. To this purpose, by writing $\lambda=\mathrm{e}^{2 \pi \mathrm{i} \alpha}$, the following Diophantine conditions were introduced. For some $\gamma>0$ and $\tau>0$ it is required that

$$
\left|\alpha-\frac{p}{q}\right| \geq \frac{\gamma}{q^{\tau}}
$$


for all rationals $p / q$ (with $q>0$ ). It turns out that this is sufficient for convergence of the formal solution for $\Phi$.

Let us discuss certain aspects of the set of $\lambda \in \mathbf{T}^{1}$ satisfying the Diophantine conditions, as these are relevant for us. It can be directly shown that for fixed $\gamma>0$ and $\tau>2$, with $\gamma$ sufficiently small, the Diophantine conditions give rise to a Cantor subset $\lambda \in \mathbf{T}^{1}$ which is of almost full measure. We recall that the characterizing (topological) properties of a Cantor set are compactness, nowhere denseness and perfectness. It can be easily seen that the measure of the complement is of order $O(\gamma)$ as $\gamma \downarrow 0$. Compare with, e.g., [17], [23. We note that for small values of $\gamma$ (as well as for large values of $\tau$ ) only a small radius of convergence for $\Phi$ can be guaranteed [65].

Geometry and number theory. That the Diophantine conditions give rise to a nowhere dense subset of $\mathbf{T}^{1}$ directly follows from the fact that the roots of unity, which are everywhere dense, are in their complement. The following example, due to H. Cremer [24] in 1927, already indicates that there is more to this. For a nice description of this example in a somewhat different context, see [10].

Consider the map $F: \mathbf{C} \rightarrow \mathbf{C}$ given by

$$
F(z)=\lambda z+z^{2},
$$

where $\lambda \in \mathbf{T}^{1}$ is not a root of unity. We shall see that there is a topologically large subset of $\lambda$ for which the map has periodic points in any neighbourhood of 0 . For such $\lambda$ this provides a geometrical proof that the formal conjugation diverges, as follows. Indeed, since the linear map $z \mapsto \lambda z$ is an irrational rotation, all of whose orbits are everywhere dense in $\mathbf{T}^{1}$, the existence of periodic orbits in every neighbourhood of 0 implies that the formal linearization must have zero radius of convergence.

To examine periodic points of period $q$, we consider the equation

$$
F^{q}(z)=z .
$$

Using that

$$
F^{q}(z)=\lambda^{q} z+\cdots+z^{2^{q}}
$$

it follows that

$$
F^{q}(z)-z=z\left(\lambda^{q}-1+\cdots+z^{2^{q}-1}\right) .
$$

Abbreviating $N=2^{q}-1$, let $z_{1} \cdot z_{2}, \cdots, z_{N}$ be the nontrivial solutions of (3). It follows that their product satisfies the equation

$$
z_{1} \cdot z_{2} \cdot \cdots \cdot z_{N}=\lambda^{q}-1
$$

From this we see that there exists a solution within radius

$$
\left|\lambda^{q}-1\right|^{1 / N}
$$

of $z=0$. Now consider the set of $\lambda \in \mathbf{T}^{1}$ satisfying

$$
\liminf _{q \rightarrow \infty}\left|\lambda^{q}-1\right|^{1 / N}=0 .
$$

This set turns out to be residual 1 We recall that a residual set contains a countable intersection of dense-open sets; this property means that the set is large in the sense of topology. The present residual set resembles that of the Liouville-numbers. Notice that our residual set has measure zero, since it has all Diophantine numbers

\footnotetext{
${ }^{1}$ In other words a dense $G_{\delta}$ or a set of 2 nd Baire category.
} 
in its complement. For a general background reference dealing with subsets of $\mathbf{R}$ that are large in topology and small in measure, or vice versa, see Oxtoby [56].

We conclude that for all $\lambda$ contained in this residual set, periodic points of $F$ occur in any neighbourhood of $z=0$, which makes the above divergence argument work.

By the end of the 20th century J.-C. Yoccoz 68, 69 completely solved the elliptic case, proving that the so-called Bruno-condition [20] on the corresponding continued fractions is necessary and sufficient for convergence of the formal linearization for all possible higher order terms.

\section{Circle maps}

We slightly shift context by considering a smooth (for definiteness, say $C^{\infty}$ or real analytic) family of circle diffeomorphisms

$$
P_{\alpha, \varepsilon}: \mathbf{T}^{1} \rightarrow \mathbf{T}^{1} ; x \mapsto x+2 \pi \alpha+\varepsilon a(x, \alpha, \varepsilon),
$$

where $\varepsilon$ is a small parameter, so we are in the neighbourhood of a family of rigid circle rotations. For convenience we rewrite this as a 'vertical' family of cylinder maps

$$
P_{\varepsilon}: \mathbf{T}^{1} \times[0,1] \rightarrow \mathbf{T}^{1} \times[0,1] ;(x, \alpha) \mapsto(x+2 \pi \alpha+\varepsilon a(x, \alpha, \varepsilon), \alpha) .
$$

The problem is to find a diffeomorphism $\Phi_{\varepsilon}$ that conjugates $P_{\varepsilon}$ and $P_{0}$. Note that the latter map is associated to the family of rigid rotations. To be precise, we require that the following diagram commutes:

$$
\begin{array}{cccc}
\mathbf{T}^{1} \times[0,1] & \stackrel{P_{\varepsilon}}{\longrightarrow} & \mathbf{T}^{1} \times[0,1] \\
\uparrow \Phi_{\varepsilon} & & \uparrow \Phi_{\varepsilon} \\
\mathbf{T}^{1} \times[0,1] & \stackrel{P_{0}}{\longrightarrow} & \mathbf{T}^{1} \times[0,1],
\end{array}
$$

meaning that

$$
P_{\varepsilon} \circ \Phi_{\varepsilon}=\Phi_{\varepsilon} \circ P_{0} .
$$

Small divisors again. We proceed by formally solving equation (44). Indeed, assuming that $\Phi_{\varepsilon}$ has the form

$$
\Phi_{\varepsilon}(x, \alpha)=(x+\varepsilon U(x, \alpha, \varepsilon), \alpha+\varepsilon \sigma(\alpha, \varepsilon))
$$

it follows that (4) can be rewritten as

$$
U(x+2 \pi \alpha, \alpha, \varepsilon)-U(x, \alpha, \varepsilon)=2 \pi \sigma(\alpha, \varepsilon)+a(x+\varepsilon U(x, \alpha, \varepsilon), \alpha+\varepsilon \sigma(\alpha, \varepsilon), \varepsilon) .
$$

Expanding in powers of $\varepsilon$ and comparing lowest order coefficients, equation (4) leads to the linear equation

$$
U_{0}(x+2 \pi \alpha, \alpha)-U_{0}(x, \alpha)=2 \pi \sigma_{0}(\alpha)+a_{0}(x, \alpha) .
$$

This linear equation can be directly solved by Fourier-series. Indeed, writing

$$
\begin{aligned}
a_{0}(x, \alpha) & =\sum_{k \in \mathbf{Z}} a_{0 k}(\alpha) \mathrm{e}^{\mathrm{i} k x}, \\
U_{0}(x, \alpha) & =\sum_{k \in \mathbf{Z}} U_{0 k}(\alpha) \mathrm{e}^{\mathrm{i} k x},
\end{aligned}
$$


we find that $\sigma_{0}=-\frac{1}{2 \pi} a_{00}$, which amounts to a parameter shift, and that

$$
U_{0 k}(\alpha)=\frac{a_{0 k}(\alpha)}{\mathrm{e}^{2 \pi \mathrm{i} k \alpha}-1} .
$$

Similar to what we had in the previous case, we observe that there exists a formal solution as soon as $\alpha$ is irrational. As before, the powers of $\mathrm{e}^{2 \pi \mathrm{i} \alpha}$ may still accumulate on 1 . This gives small divisors in the Fourier series, which puts its convergence in doubt.

A KAM theorem for circle maps. To overcome the problem of small divisors, we introduce the same Diophantine conditions as before, requiring that for given numbers $\tau>2$ and $\gamma>0$ and for all rationals $p / q$, one has

$$
\left|\alpha-\frac{p}{q}\right| \geq \frac{\gamma}{q^{\tau}}
$$

In correspondence to what was said earlier, this gives a Cantor set $[0,1]_{c} \subseteq[0,1]$, of almost full measure as $\gamma>0$ is small. As our first example of an actual KAM theorem we now formulate

Theorem 1. In the above circumstances assume that $\gamma>0$ is sufficiently small. Then, for $|\varepsilon|$ sufficiently small, there exists a smooth $\left(C^{\infty}\right)$ transformation of the cylinder $\Phi_{\varepsilon}: \mathbf{T}^{1} \times[0,1] \rightarrow \mathbf{T}^{1} \times[0,1]$, conjugating the restriction $\left.P_{0}\right|_{\mathbf{T}^{1} \times[0,1]_{c}}$ to a subsystem of $P_{\varepsilon}$. Moreover, $\Phi$ depends smoothly on $\varepsilon$.

This result goes back to V.I. Arnold [4]; also compare with [5]. The present formulation closely fits with [18, [17. Concerning the smoothness of the map $\Phi_{\varepsilon}$, compare with Pöschel [57] and with [66], [70, [71]. The actual proof of Theorem 1 does not deal with the power series in $\varepsilon$. Instead it uses a Newton method and an approximation property by analytic maps of Whitney smooth maps defined on closed sets.

We observe that KAM Theorem 1 has a strongly perturbative character: indeed it only applies to small perturbations of the rigid rotation family. In contrast to this M.R. Herman [34] and J.-C. Yoccoz [67, 68] have proven a non-perturbative version of this theorem, i.e., for large values of $\varepsilon$. Also see [48].

Discussion. A circle diffeomorphism smoothly conjugated to a rigid rotation $x \mapsto$ $x+2 \pi \alpha$ with $\alpha$ irrational is called quasi-periodic. It is not hard to show that each orbit of such a quasi-periodic map fills the circle densely [25]. Notice that for $\alpha \in[0,1]_{c}$ the map rigid rotation $P_{\alpha, 0}$ certainly is quasi-periodic.

A first consequence of Theorem 1 then is that the circle maps $P_{\alpha, \varepsilon}$ that are conjugated to one of the Diophantine - and hence quasi-periodic - rotations $P_{\alpha, 0}$ are still quasi-periodic. So we conclude that quasi-periodicity typically occurs with positive measure in the parameter space. Moreover, the fact that a Cantor set is perfect 2 implies that in this setting quasi-periodicity never occurs as an isolated phenomenon.

As a concrete example consider the Arnold family

$$
P_{\alpha, \varepsilon}(x)=x+2 \pi \alpha+\varepsilon \sin x
$$

of circle maps, looking at the $(\alpha, \varepsilon)$-plane of parameters. We restrict to $|\varepsilon|<1$ to ensure that $P_{\varepsilon}$ is a diffeomorphism. It is known [4, [5], 25] that from the points

\footnotetext{
${ }^{2}$ Which means that it contains no isolated points.
} 
$(\alpha, \varepsilon)=(p / q, 0)$ resonance tongues emanate into the open half-planes $\varepsilon \neq 0$ in such a way that for small $|\varepsilon|$ an open and dense subset is covered. In the tongue emanating from $(\alpha, \varepsilon)=(p / q, 0)$ the dynamics is periodic, with rotation number $p / q$ (for a definition see, e.g., [25]).

Theorem 1 implies that in the complement of this union of tongues there exists a union of smooth curves that fills out positive measure. For parameter values on these curves, the dynamics is quasi-periodic.

Theorem 1 has a number of applications. Indeed, it applies whenever a system of ordinary differential equations has a 2-torus attractor, of which $P_{\varepsilon}$ is a Poincaré map close to a rigid rotation family. In that case the quasi-periodic subsystem of $P_{\varepsilon}$, for $|\varepsilon| \ll 1$, often is referred to as a family of quasi-periodic attractors [61.

Also in the general case resonance tongues are (generically) present, and we find the same intricate interplay of periodicity and quasi-periodicity as in the Arnold family.

Higher dimensional analogues of the present situation exist, where next to periodicity and quasi-periodicity chaotic dynamics also coexists [18, [17. This scenario and the transitions or bifurcations between the various kinds of dynamics have been associated to the onset of turbulence in fluid dynamics; see Ruelle and Takens et al. 62, 55]. The quasi-periodic state then is seen as intermediate between very orderly and chaotic. Also see [35], 43], [44].

\section{THE TWIST THEOREM}

Returning to the conservative setting we consider an annulus $A \subseteq \mathbf{R}^{2}$ with 'polar' coordinates $(\varphi, I) \in \mathbf{T}^{1} \times \mathbf{K}$, where $\mathbf{K}$ is compact. We endow $A$ with the area form $\sigma=d \varphi \wedge d I$. Consider a smooth (say $C^{\infty}$ ) map $P_{\varepsilon}: A \rightarrow A$ of the form

$$
P_{\varepsilon}(\varphi, I)=(\varphi+2 \pi \alpha(I), I)+O(\varepsilon)
$$

that preserves the area $\sigma$. Note that for $\epsilon=0$ the map leaves the family of circles $I=$ cst invariant, and again the problem is the persistence of this family for $|\varepsilon|$ small. We say that the map $P_{0}$ is a (pure) twist map if the map $I \mapsto \alpha(I)$ is a diffeomorphism.

We assume Diophantine conditions as before: For given constants $\tau>2$ and $\gamma>0$ let

$$
\left|\alpha-\frac{p}{q}\right| \geq \frac{\gamma}{q^{\tau}}
$$

for all rationals $p / q$. Via the map $\alpha: I \mapsto \alpha(I)$ this set is pulled back to a subset $A_{c} \subseteq A$, which is the product of a circle and a Cantor set of large measure.

Theorem 2. In the above circumstances assume that $\gamma>0$ is sufficiently small. Then for $|\varepsilon|$ sufficiently small, there exists a smooth $\left(C^{\infty}\right)$ transformation of the annulus $\Phi_{\varepsilon}: A \rightarrow A$, conjugating the restriction $\left.P_{0}\right|_{A_{c}}$ to a subsystem of $P_{\varepsilon}$. Moreover, $\Phi$ depends smoothly on $\varepsilon$.

This theorem is known as the 'twist' theorem, and it was first proven by J.K. Moser [49]. The present formulation is close to Theorem 1, and concerning the smoothness of $\Phi_{\varepsilon}$, the same comments apply again here.

Discussion. Regarding the similarity between Theorems 1 and 2 one can say that in Theorem 2 the rôle of the parameter $\alpha$ has been taken by the action variable $I$. 
In the same spirit as before, we conclude that for the area preserving case, typically quasi-periodicity occurs with positive measure in phase space.

Remark. A difference in the settings of Theorems 1 and 2 is the following. In the case of KAM Theorem 1 for circle maps, the conjugation $\Phi_{\varepsilon}$ between the family of pure rotations $P_{0}$ and the perturbation $P_{\varepsilon}$ preserves the projection to the parameter space $[0,1]=\{\alpha\}$; compare with equation (5). In the present case of Theorem 2 the corresponding projection to the action space $K=\{I\}$ generally is not preserved.

As an application consider the mathematical (planar) pendulum with (weak) periodic forcing. As possible equations of motion one may take

$$
\begin{array}{rlr}
\ddot{y}+\omega^{2} \sin y & =\varepsilon \cos t \quad \text { or } \\
\ddot{y}+\left(\omega^{2}+\varepsilon \cos t\right) \sin y & =0,
\end{array}
$$

which give rise to volume-preserving 3-dimensional vector fields. For simplicity we write down only the first example:

$$
\begin{aligned}
\dot{y} & =z \\
\dot{z} & =-\omega^{2} \sin y+\varepsilon \cos t \\
\dot{t} & =1 .
\end{aligned}
$$

As is common in mechanics (e.g., see [6]), we introduce action-angle variables $(I, \varphi)$ for $\varepsilon=0$, i.e., for the autonomous planar pendulum. In fact, denoting the energy by $H_{0}(y, z)=\frac{1}{2} z^{2}-\omega^{2} \cos y$, we restrict ourselves to the oscillatory region where $H_{0}(y, z)<\omega^{2}$. Next consider any level set $H_{0}(y, z)=h$, with $|h|<\omega^{2}$. The action variable $I$ then is defined by

$$
I(h)=\frac{1}{2 \pi} \oint_{H_{0}(y, z)=h} z d y,
$$

which is proportional to the area enclosed by the level set. The angle variable $\varphi$ is obtained by taking the time parametrization of the periodic motion inside this level set scaled to period $2 \pi$. Thus one obtains the canonical equations

$$
\dot{I}=0, \dot{\varphi}=\alpha(I)
$$

for the oscillatory motions of the planar pendulum.

This implies that the Poincaré (or stroboscopic) map $P_{\varepsilon}$ has the above form (6). A direct computation $3^{3}$ shows that $P_{0}$ is a pure twist map. Therefore the conclusion of quasi-periodicity occurring with positive measure in phase space applies here.

A related application deals with coupled oscillators

$$
\begin{aligned}
& \ddot{y}_{1}=-\omega_{1}^{2} \sin y_{1}-\varepsilon \frac{\partial U}{\partial y_{1}}\left(y_{1}, y_{2}\right) \\
& \ddot{y}_{2}=-\omega_{2}^{2} \sin y_{2}-\varepsilon \frac{\partial U}{\partial y_{2}}\left(y_{1}, y_{2}\right),
\end{aligned}
$$

leading to a 4-dimensional Hamiltonian vector field

$$
\begin{aligned}
& \dot{y}_{j}=z_{j} \\
& \dot{z}_{j}=-\omega_{j}^{2} \sin y_{1}-\varepsilon \frac{\partial U}{\partial y_{j}}\left(y_{1}, y_{2}\right),
\end{aligned}
$$

${ }^{3}$ Involving an elliptic integral ... 
$j=1,2$, with Hamilton function $H_{\varepsilon}\left(y_{1}, z_{1}, y_{2}, z_{2}\right)=\frac{1}{2} z_{1}^{2}+\frac{1}{2} z_{2}^{2}-\omega_{1} \cos y_{1}-$ $\omega_{2} \cos y_{2}+\varepsilon U\left(y_{1}, y_{2}\right)$. In this case it is the iso-energetic Poincaré map which obtains the form $P_{\varepsilon}$. This leads to the conclusion of quasi-periodicity occurring with positive measure in energy hypersurfaces of $H_{\varepsilon}$.

\section{Conservative KAM theory}

The above discussion has set the stage for a general formulation of the KAM theorem as a suitable variation on Kolmogorov [39], 40] and Arnold [1.

We begin by introducing the notion of a quasi-periodic invariant torus for an autonomous system of ordinary differential equations. Indeed, we understand this to be an invariant torus, the dynamics on which is smoothly conjugated to that of a constant vector field

$$
\begin{aligned}
& \dot{x}_{1}=\omega_{1} \\
& \dot{x}_{2}=\omega_{2} \\
& \cdots \\
& \dot{x}_{n}=\omega_{n},
\end{aligned}
$$

on the standard $n$-torus $\mathbf{T}^{n}=\mathbf{R}^{n} /\left(2 \pi \mathbf{Z}^{n}\right)=\left\{x_{1}, x_{2}, \ldots, x_{n}\right\}$, where the angles $x_{j}$ are counted modulo $2 \pi, 1 \leq j \leq n$. Moreover, the frequencies $\omega_{1}, \omega_{2}, \ldots, \omega_{n}$ are required to be independent over the rationals, which implies that the torus is densely filled by each of its trajectories. In that case we speak of $n$ quasi-periodicity, both for the restriction of the system to the $n$-torus as for the $n$-torus itself.

For integrable Hamiltonian systems, the conjugation with a constant vector field is provided by the action angle variables provided by the Liouville-Arnold theorem [6].

The present formulation of the classical KAM theorem now reads

Theorem 3. In Hamiltonian systems with $n$ degrees of freedom typically $n$ quasiperiodic Lagrangean tori occur with positive Liouville measure, both

(1) in phase space

(2) and in each energy hypersurface.

As said earlier 'typical' means that there are classes of examples that are 'open in function space'. These examples are close to Liouville integrable systems [6].

The first conclusion of Theorem 3 is the standard KAM theorem, while the second is referred to as the iso-energetic KAM theorem. The nondegeneracy condition on the integrable approximation $H=H(I)$ is somewhat different for both cases.

Indeed, for the standard KAM theorem the Kolmogorov nondegeneracy condition is required, which states that the frequency vector

$$
\omega=\frac{\partial H}{\partial I}
$$

as a function of the action variables $I$ should have maximal rank; this implies that the frequency map $I \mapsto \omega(I)$ locally is a diffeomorphism.

The condition for iso-energetic nondegeneracy similarly requires that the corresponding frequency ratio map $I \mapsto\left(\omega_{1}(I): \omega_{2}(I): \ldots: \omega_{n}(I)\right)$ to the $(n-1)$ dimensional projective space should have maximal rank. Compare with [6], [18], [15]. We remark that the conclusion drawn in the example of the two coupled oscillators of the previous section also would follow as an application of the iso-energetic KAM theorem. 
In typical cases, however, both nondegeneracy conditions are satisfied, implying that the union of quasi-periodic Lagrangean invariant tori has positive measure in phase space in such a way that the conditional measure in the energy hypersurfaces also is positive.

In addition to nondegeneracy one again needs Diophantine conditions on the frequencies, in this case defined as follows. For constants $\tau>n-1$ and $\gamma>0$, it is required that for all $k \in \mathbf{Z}^{n} \backslash\{0\}$ one has

$$
|\langle\omega, k\rangle| \geq \gamma|k|^{-\tau} .
$$

Here $\langle\omega, k\rangle=\sum_{j=1}^{n} \omega_{j} k_{j}$, while $|k|=\sum_{j=1}^{n}\left|k_{j}\right|$. Similar to the cases met before, the corresponding subset of $\mathbf{R}^{n}$ is nowhere dense of positive measure. In fact this Diophantine subset is a 'bundle' of closed half-lines, the intersection of which with the unit sphere $\mathbf{S}^{n-1}$, for small $\gamma$, contains a Cantor set of almost full measure. Compare with, e.g., [23, [17.

In the case of the general Kolmogorov nondegeneracy, this set is pulled back along the frequency map in a locally diffeomorphic way. Iso-energetic nondegeneracy means that the energy hypersurfaces are transversal to the Diophantine line bundle; compare with [18, [15.

Some applications of the KAM theorem. In the introduction we already mentioned possible applications of Theorem 3. Indeed, in many models of classical mechanics one finds Lagrangean KAM tori with quasi-periodic dynamics.

As a 'philosophical' application consider the solar system, first taken as a perturbation of the integrable system obtained when neglecting the interaction between the planets. If the theorem were to apply, it would follow that the actual evolution has positive probability to be quasi-periodic, when assuming that the initial condition has been chosen at random. In that case one might be inclined to call the solar system stable.

Much has been said about this example [2], [52, [53], 63], 26, and here we just restrict ourselves to a few remarks. Firstly the solar system contains quite strong resonances, which necessitate a more suitable integrable approximation than described here [2]. Secondly the interaction between the planets probably is far too strong for an actual application of Theorem 3 as a perturbation result. The third remark refers to recent numerical work of Laskar [45], which seems to show that the solar system is entirely chaotic; mankind just has not existed long enough to have noticed ....

A large class of examples is provided by considering the neighbourhood of any generic elliptic equilibrium point of a Hamiltonian system. Under a few nonresonance conditions on the eigenvalues of the linear part one can normalize a number of steps according to Birkhoff, thereby making the lower order terms $n$-torus symmetric. In fact this allows writing the system locally as a nearly integrable system, where the size of the perturbation is controlled by the distance to the equilibrium; compare 52, 53.

As a consequence, in a neighbourhood of the equilibrium there are many KAM tori. Their union is of positive Liouville measure, where the equilibrium point is even a density point of quasi-periodicity [57, [17].

Other applications of KAM-techiques occur in quantum mechanics, in particular in the study of electron - Anderson - localization. If one considers Schrödinger equations with spatially ergodic potentials, a localized (non-conducting) state is an 
eigenfunction of the Schrödinger equation at some energy eigenvalue. Such a function will decay exponentially in space. As in the localized regime the Schrödinger operator typically has a dense point spectrum, so developing a perturbation theory of the corresponding resolvent operator, which diverges at this dense set of eigenvalues, runs into problems very similar to problems encountered in the traditional KAM set-up, where the dense set of resonances gives rise to divergent perturbation expansions. KAM-inspired proofs and analyses of localization have been developed for typical realizations of random potentials in arbitrary dimensions for large interaction strengths or high energies in arbitrary dimensions (see e.g. 29]), as well as for quasi-periodic potentials in one dimension, describing electrons in quasicrystals; see e.g. [27], [19], 60].

Yet another field of physics which is notorious for divergent perturbation theory problems and where KAM-like ideas are starting to play a role is Quantum Field Theory [32], 9 .

Discussion. There are a great many related issues spread over a vast literature compare with [6], [7, [52], [53, 63], 26] - and we will be restrictive here.

One subject to mention is the difference between the cases $n=2$ and $n \geq 3$. Indeed, for $n=2$ the level set of the energy function is 3 dimensional and the Lagrangean tori have dimension 2. In the nearly integrable case these KAM tori generally foliate over nowhere dense sets. However, for open sets of initial conditions the evolution curves are forever trapped in between them, which implies perpetual adiabatic stability.

In contrast, for $n \geq 3$ the Lagrangean tori have codimension larger than 1 and evolution curves may escape. This actually occurs in case of Arnold diffusion [3], [7], [21].

Another subject concerns the general motion in the neighbourhood of the KAM tori. Indeed by refined averaging it follows that nearby evolution curves stay nearby over an exponentially long time; see Nekhoroshev [54]. Colloquially one says that the KAM tori are 'sticky'.

From the first developments of KAM theory on, it was known that the theory extends outside the Hamiltonian frame work of Lagrangean tori. So it turns out that analogues of Theorem 3 exist, e.g., for lower dimensional (isotropic) invariant tori in Hamiltonian systems. Here, apart from the internal frequencies of the quasiperiodic tori, normal frequencies also play a role, and these moreover enter the Diophantine conditions. For a discussion on the dynamical role of the corresponding normal-internal resonances, see [12.

There has been quite a lot of interest in the Hamiltonian KAM theory of normally elliptic tori [58, 37]. This also includes cases in which the normal frequencies have strong resonances [36. Moreover, cases have been studied of normally parabolic tori. The latter two cases involve quasi-periodic torus bifurcations, in certain cases also branching off higher dimension tori [17, [33, 13], 14.

Similar results occur when considering reversible systems instead of Hamiltonian ones [50, [53, 64], 16], 22], or systems that are equivariant with respect to certain Lie groups. Moreover, as section 3 already indicates, there also exist KAM theorems for the general class of (dissipative) dynamical systems; compare with section 3 . In fact a unifying Lie algebra approach allows us to reach all these results at once. In general one needs external parameters to have a good persistence theory for quasi-periodic invariant tori. Compare with [50], [51], [18], [17]. 


\section{ERGODIC HYPOTHESIS: A PARADOX?}

As a direct corollary of Theorem 3 we have

Theorem 4. In typical Hamiltonian systems with $n<\infty$ degrees of freedom, energy hypersurfaces are not ergodic under the Hamiltonian flow.

Indeed, by Theorem 3 there exist open classes of Hamiltonian systems with invariant tori such that the union of these tori has positive (Liouville) measure in the energy hypersurfaces. This leads to a decomposition of these hypersurfaces in several disjoint invariant sets, which contradicts ergodicity. For background on ergodic theory, e.g., see [7].

So the KAM tori form an obstruction against ergodicity, and a question is how bad this obstruction is as $n \rightarrow \infty$. This question is of importance in statistical physics, where large systems are studied.

A primitive formulation of the ergodic hypothesis asserts that typically, energy hypersurfaces of many-particle systems are ergodic, which clearly would not be compatible with Theorem 4 . To fix thoughts we give an example.

A lattice system. Consider the 1-dimensional lattice $\mathbf{Z} \subset \mathbf{R}$, at the vertices of which identical nonlinear oscillators are situated. For simplicity think of the lattice being situated on a horizontal line where at all vertices identical pendula are suspended, subject to constant vertical gravity. Also we connect nearest neighbour oscillators by weak springs. Here the spring constants can be either constant or decay at infinity.

Let $\Lambda_{N} \subseteq \mathbf{Z}$ be the box with vertices $(-N, N)$. Then, for $M \leq N$ consider two of these boxes, $\Lambda_{M} \subseteq \Lambda_{N}$. We 'truncate' the infinite system by ignoring all pendula outside the larger box $\Lambda_{N}$.

First consider the integrable system associated to $\Lambda_{N}$, where all interactions are neglected. Suppose that the oscillators situated at vertices in $\Lambda_{M}$ are in motion while the others are at rest. In the phase space this corresponds to a $2 M+1$ dimensional invariant torus, which is normally elliptic. Moreover, the normal frequencies are in $1: 1: \ldots: 1$ resonance.

Then we 'turn on' the activity of the interacting springs. A suitable adaptation [36] of Theorem 3 and of [58] for this case yields the persistence of these elliptic tori for small values of the spring constants. The corresponding kind of motion is a quasi-periodic breather; for a similar kind of motion compare with 47. The union of elliptic tori has positive $2(2 M+1)$-dimensional Hausdorff measure.

The question now is, what is the asymptotics of the 'density' of this measure as $N$ (and $M) \rightarrow \infty$ ? A partial answer to this question [36] says that this density decays exponentially fast in $N$, while there is a further polynomial decay in $M$.

Remark. It is worth mentioning that the normal $1: 1: \ldots: 1$ resonance of this system gives rise to interesting quasi-periodic branchings [36].

Discussion. The conclusion from this example is that the obstruction to ergodicity given by KAM theory is not too bad as the size of the system tends to infinity. This is along the same lines as an earlier result by Arnold [3].

Another problem is what happens if the limit $N \rightarrow \infty$ is really attained. The KAM theory for infinite systems is fully in development [41], [38], but infinite lattice systems still have many secrets. 


\section{Conclusion}

The lasting influence of Kolmogorov, Arnold and Moser on the present state of the art in mathematics, physics and other sciences is enormous, and this paper sketches only part of this legacy. Nevertheless we believe that it is an important part, which is still fully in development. The role of the ergodic hypothesis in statistical mechanics has turned out to be much more subtle than was expected; see e.g., 8, 30, 31. Regarding KAM theory, for further reading we mention the introductory texts [23, 48], [46], [59]. Also, reading of [17], 42] and [28] is recommended.

The author thanks the Universitat de Barcelona and the Université de Bourgogne for hospitality during the preparation of this paper. Also I thank Aernout van Enter and Floris Takens for helpful discussions.

\section{REFERENCES}

[1] V.I. Arnold, Proof of a theorem by A.N. Kolmogorov on the invariance of quasi-periodic motions under small perturbations of the Hamiltonian, Usp. Math. Nauk 18(5) (1963) 13-40; Russ. Math. Surv. 18(5) (1963) 9-36. MR 29:328

[2] V.I. Arnold, Small denominators and problems of stability of motion in classical and celestial mechanics, Russian Math. Surveys 18(6) (1963) 85-191. [Corrigenda (in Russian): Uspekhi Mat. Nauk 23 (1968) 216.] MR 30:943

[3] V.I. Arnold, Instability of dynamical systems with many degrees of freedom, Soviet Mathematics 5(3) (1964) 581-585. MR 29:329.

[4] V.I. Arnold, Small divisors I: On mappings of the circle onto itself, Amer. Math. Soc. Transl., Ser.2 46 (1965) 213-284. [Russian original: Izvest. Akad. Nauk SSSR, Ser Mat. 25(1) (1961) 21-86; Corrigenda: ibid. 28(2) (1964) 479-480.] MR 25:4113

[5] V.I. Arnold, Geometrical Methods in the Theory of Ordinary Differential Equations. Springer Verlag 1988. [Russian original: Nauka 1978.] MR 89h:58049]

[6] V.I. Arnold, Mathematical Methods in Classical Mechanics. Springer Verlag 1978, 2nd edition 1989. [Russian original: Nauka 1974.] MR 57:14033b, MR 90c:58046

[7] V.I. Arnold and A. Avez, Ergodic Problems of Classical Mechanics. Addison-Wesley 1989. [French original: Gauthier-Villars 1968.] MR 38:1233

[8] J. Bricmont, Science of Chaos or Chaos in Science? In P.R. Gross, N. Levitt and M.W. Lewis (eds.), The Flight from Science and Reason, Ann. of the New York Academy of Sciences 775 131-175, New York Academy of Sciences, New York 1996. [Also appeared in Physicalia Magazine 17 (1995) 159-208.] MR 99h:00012

[9] J. Bricmont, K. Gawedzki and A. Kupiainen, KAM Theorem and Quantum Field Theory, Comm. Math. Phys. 201 (1999) 699-727. MR 2000d:37070

[10] P. Blanchard, Complex analytic dynamics on the Riemann sphere, Bull. Amer. Math. Soc. (N.S.) 11(1) (1984) 85-141. MR 85h:58001

[11] H.W. Broer, A.N. Kolmogorov: la 'K' de KAM, Bull. de la Societat Catalana de Matemàtiques (2004), to appear.

[12] H.W. Broer, H. Hanßmann, À. Jorba, J. Villanueva and F.O.O. Wagener, Normal-internal resonances in quasiperiodically forced oscillators: a conservative approach, Nonlinearity $\mathbf{1 6}$ (2003) 1751-1791.

[13] H.W. Broer, H. Hanßmann and J. You, Bifurcations of normally parabolic tori in Hamiltonian systems, Preprint University of Groningen. Submitted for publication.

[14] H.W. Broer, H. Hanßmann and J. You, Umbilical torus bifurcations in Hamiltonian systems, Preprint University of Groningen. Submitted for publication.

[15] H.W. Broer and G.B. Huitema, A proof of the iso-energetic KAM-theorem from the 'ordinary' one, Journ. Diff. Eqns. 90(1) (1991) 52-60. MR 92a:58117

[16] H.W. Broer and G.B. Huitema, Unfoldings of quasi-periodic tori in reversible systems, Journ. Dynamics and Differential Equations 7(1) (1995) 191-212. MR 96b:58099

[17] H.W. Broer, G.B. Huitema and M.B. Sevryuk, Quasi-periodic motions in families of dynamical systems: order amidst chaos, LNM 1645. Springer Verlag 1996. MR 99d:58142 
[18] H.W. Broer, G.B. Huitema, F. Takens and B.L.J. Braaksma, Unfoldings and bifurcations of quasi-periodic tori, Mem. AMS 83(421) (1990) 1-170. MR 91e:58156

[19] H.W. Broer, J. Puig and C. Simó, Resonance tongues and instability pockets in the quasiperiodic Hill-Schrödinger equation, Commun. Math. Phys. 641 (2003) 467-503.

[20] A.D. Bruno, Convergence of transformations of differential equations to the normal forms, Dokl. Akad. Nauk. SSSR. 165 (1965) 987-989. MR 33:325

[21] L. Chierchia and G. Gallavotti, Drift and diffusion in phase space, Ann. Inst. Henri Poincaré, Physique Théorique 60(1) (1994) 1-144. MR 95b:58056

[22] M.C. Ciocci, Bifurcation of periodic orbits and persistence of quasi periodic solutions in families of reversible systems. Ph.D. Thesis, Gent 2003.

[23] M.C. Ciocci, A. Litvak-Hinenzon and H.W. Broer, Survey on dissipative KAM theory including quasi-periodic bifurcations, based on lectures by Henk Broer. To appear in J. Montaldi and T. Ratiu (eds.): Peyresq Lectures on Geometric Mechanics and Symmetry, LMS Lecture Notes Series 306. Cambridge University Press.

[24] H. Cremer, Zum Zentrumproblem, Math. Ann. 98 (1927) 151-163.

[25] R.L. Devaney, An Introduction to Chaotic Dynamical Systems. Addison Wesley 1989. MR 91a:58114

[26] F. Diacu and P. Holmes, Celestial Encounters, the origins of chaos and stability. Princeton University Press 1996. MR 98e:70006

[27] E.I. Dinaburg and Y.G. Sinai, The one-dimensional Schrödinger equation with a quasiperiodic potential. Func. Anal. and Appl. 9 (1975) 179-189. MR 57:10076

[28] L.H. Eliasson, S.B. Kuksin, S. Marmi and J.-C. Yoccoz, Dynamical Systems and Small Divisors, Cetraro 1998, LNM 1784. Springer Verlag 2002. MR 2003c:37001

[29] J. Fröhlich and T. Spencer, Absence of diffusion in the Anderson model for large disorder or low energy, Comm. Math. Phys. 88 (1983) 151-189. MR 85c:82004

[30] G. Gallavotti, Statistical Mechanics, A Short Treatise. Springer Verlag 1999. MR 2001j:82001

[31] G. Gallavotti, F. Bonetto and G. Gentile, Aspects of the ergodic, qualitative and statistical theory of motion. To appear Springer Verlag. Preliminary version at http://ipparco.roma1.infn.it/libri.html.

[32] G. Gallavotti, G. Gentile and V. Mastropietro, Field theory and KAM tori, MPEJ 1 (1995) paper 5. MR 97b:81072

[33] H. Hanßmann, The quasi-periodic centre-saddle bifurcation, Journ. Diff. Eqns. 142(2) (1998) 305-370. MR 98m:58099

[34] M.R. Herman, Sur la conjugaison différentiable des difféomorphismes du cercle à des rotations, Publ. Math. IHES 49 (1979) 5-234. MR 81h:58039

[35] E. Hopf, A mathematical example displaying features of turbulence. Commun. Appl. Math. 1 (1948) 303-322. MR 10:716a

[36] H.H. de Jong, Quasiperiodic breathers in systems of weakly coupled pendulums: Applications of KAM theory to classical and statistical mechanics. Ph.D. Thesis, Groningen 1999.

[37] A. Jorba and J. Villanueva, On the normal behaviour of partially elliptic lower-dimensional tori of Hamiltonian systems, Nonlinearity 10 (1997) 783-822. MR 98h:58169

[38] T. Kappeler and J. Pöschel, KdV \& KAM, Ergebnisse der Mathematik und ihrer Grenzgebiete, 3. Folge 45. Springer Verlag 2003.

[39] A.N. Kolmogorov, On the conservation of conditionally periodic motions for a small change in Hamilton's function [in Russian], Dokl. Akad. Nauk SSSR 98 (1954) 525-530; English translation in LNP 93 (1979) 51-56. MR 16:924c

[40] A.N. Kolmogorov, The general theory of dynamical systems and classical mechanics. Proceedings of the International Congress of Mathematicians (Amsterdam, 1954), Vol. 1, pages 315-333, North Holland, Amsterdam, 1957 [in Russian]. [Reprinted in: International Mathematical Congress in Amsterdam, 1954 (Plenary Lectures), pages 187-208. Fizmatgiz, Moscow 1961; English translation as Appendix D in R.H. Abraham, Foundations of Mechanics, pages 263-279. Benjamin, 1967. Reprinted as Appendix in R.H. Abraham and J.E. Marsden, Foundations of Mechanics, Second Edition, pages 741-757. Benjamin / Cummings 1978.] MR 20:4066

[41] S.B. Kuksin, Nearly integrable infinite-dimensional Hamiltonian systems, LNM 1556 Springer Verlag 1993. MR 95k:58145 
[42] S.B. Kuksin, V.F. Lazutkin and J. Pöschel (eds.), Seminar on Dynamical Systems (St. Petersburg, 1991) Birkhäuser 1994. MR 94m:58002

[43] L.D. Landau, On the problem of turbulence, Akad. Nauk. 44 (1944) 311-314. MR 6:246c

[44] L.D. Landau and E.M. Lifschitz, Fluid Mechanics. Pergamon, Oxford 1959. MR 21:6839

[45] J. Laskar, Large scale chaos and marginal stability in the solar system, Proceedings XIIth Int. Congress of Math. Phys. (Paris 1994), Int. Press Cambridge 1995. MR 96j:70015

[46] R. de la Llave, A tutorial on KAm Theory. In A. Katok et al., eds., Proceedings of Symposia in Pure Mathematics 69, Amer. Math. Soc. (2001), 175-292. MR 2002h:37123

[47] R.S. MacKay and S. Aubry, Proof of existence for breathers for time reversible or Hamiltonian networks of weakly coupled oscillators, Nonlinearity 7(6) (1994) 1623-1643. MR 96a:34085

[48] S. Marmi, An introduction to small divisor problems. Dipartimento di Matematica dell'Università di Pisa 2000.

[49] J.K. Moser, On invariant curves of area-preserving mappings of an annulus, Nachr. Akad. Wiss. Göttingen, Math.-Phys. Kl. II. 1 (1962) 1-20. MR 26:5255

[50] J.K. Moser, On the theory of quasiperiodic motions, SIAM Review 8(2) (1966) 145-172. MR 34:3013

[51] J.K. Moser, Convergent series expansions for quasi-periodic motions, Math. Ann. 169 (1967) 136-176. MR 34:7888

[52] J.K. Moser, Lectures on Hamiltonian systems, Mem. AMS 81 (1968) 1-60. MR 37:6060

[53] J.K. Moser, Stable and random motions in dynamical systems, with special emphasis to celestial mechanics, Ann. Math. Studies 77. Princeton University Press 1973. MR 56:1355

[54] N.N. Nekhoroshev, Exponential estimate on the stability time of near integrable Hamiltonian systems, Russ. Math. Surveys 32(6) (1977) 1-65. MR 58:18570

[55] S.E. Newhouse, D. Ruelle and F. Takens, Occurrence of strange Axiom A attractors near quasi-periodic flows on $\mathbb{T}^{m}, m \leq 3$, Commun. Math. Phys. 64 (1978) 35-40. MR 80f:58029

[56] J. Oxtoby, Measure and Category. Springer Verlag 1971. MR 81j:28003

[57] J. Pöschel, Integrability of Hamiltonian systems on Cantor sets, Comm. Pure Appl. Math. 35(5) (1982) 653-696. MR 84d:58039

[58] J. Pöschel, On elliptic lower dimensional tori in Hamiltonian systems, Math. Z. 202 (1989) 559-608. MR 91a:58065

[59] J. Pöschel. A lecture on the classical kAm theorem. In A. Katok et al., eds., Proceedings of Symposia in Pure Mathematics 69. Amer. Math. Soc. (2001), 707-732. MR 2002g:37081

[60] J. Puig, Cantor Spectrum for the Almost Mathieu Operator. Corollaries of localization, reducibility and duality. Preprint archive mp_arc 03-396 (2003).

[61] D. Ruelle, Elements of Differentiable Dynamics and Bifurcation Theory. Academic Press 1989. MR 90f:58048

[62] D. Ruelle and F. Takens, On the nature of turbulence, Commun. Math. Phys. 20 (1971) 167-192; 23 (1971) 343-4. MR 44:1297

[63] H. Rüssmann, Konvergente Reihenentwicklungen in der Störungstheorie der Himmelsmechanik. In K. Jacobs (ed.), Selecta Mathematica V, Heidelberger Taschenbücher 201 (1979) 93-260, Springer Verlag. MR 82j:70011

[64] M.B. Sevryuk, New results in the reversible KAm theory. In: S.B. Kuksin, V.F. Lazutkin and J. Pöschel (eds.), Seminar on Dynamical Systems (St. Petersburg, 1991) 184-199, Birkhäuser 1994. MR 96d:58120

[65] C.L. Siegel, Iteration of analytic functions, Ann. of Math. (2) 43 (1942) 607-612. MR 4:76c

[66] H. Whitney, Differentiable functions defined in closed sets, Trans. Amer. Math. Soc. 36(2) (1934) 369-387.

[67] J.-C. Yoccoz, $C^{1}$-conjugaisons des difféomorphismes du cercle. In: J. Palis (ed), Geometric Dynamics, Proceedings, Rio de Janeiro 1981, LNM 1007 (1983) 814-827. MR 85h:58102

[68] J.-C. Yoccoz, Théorème de Siegel, nombres de Bruno et polynômes quadratiques, Astérisque 231 (1995) 3-88. MR 96m:58214

[69] J.-C. Yoccoz, Analytic linearization of circle diffeomorphisms. In: S. Marmi, L.H. Eliasson, S.B. Kuksin and J.-C. Yoccoz (eds.), Dynamical Systems and Small Divisors, LNM $\mathbf{1 7 8 4}$ (2002) 125-174, Springer Verlag. 
[70] E. Zehnder, An implicit function theorem for small divisor problems, Bull. Amer. Math. Soc. 80(1) (1974) 174-179. MR 49:4019

[71] E. Zehnder, Generalized implicit function theorems with applications to some small divisor problems, I and II, Comm. Pure Appl. Math. 28(1) (1975) 91-140; 29(1) (1976) 49-111. MR 54:14001

Department of Mathematics and Computing Science, University of Groningen, Blauwborgue 3, NL-9747 AC, Groningen, The Netherlands

E-mail address: broer@math.rug.nl 